\title{
Driving mutually coupled gradient array coils in magnetic resonance imaging
}

\author{
Koray Ertan $^{1,2}$ | Soheil Taraghinia ${ }^{1,2}$ | Ergin Atalar ${ }^{1,2}$ \\ ${ }^{1}$ National Magnetic Resonance Research Center (UMRAM), Bilkent University, Bilkent, Ankara, Turkey \\ ${ }^{2}$ Department of Electrical and Electronics Engineering, Bilkent University, Bilkent, Ankara, Turkey
}

\section{Correspondence}

Ergin Atalar, National Magnetic Resonance Research Center (UMRAM) \& Aysel

Sabuncu Brain Research Center \&

Department of Electrical and Electronics

Engineering, Bilkent University, 06800

Bilkent, Ankara, Turkey.

Email: ergin.atalar@bilkent.edu.tr
Purpose: In contrast to conventional linear gradients, gradient coil arrays with arbitrary spatial dependency might experience strong mutual coupling. Although conventional gradient power amplifiers with feedback loop might compensate the effect of coupling, required voltages for the compensation are generally unknown and has to be considered beforehand to ensure that amplifier voltage limits are not exceeded. A first-order circuit model is proposed to be used as a feedforward model which enables analytical formulas of required voltages to drive the mutually coupled gradient coil arrays.

Theory and Methods: A first-order circuit model including the mutual couplings is provided to analytically calculate the input voltages and minimum achievable rise times for a given set of gradient array currents and amplifier limitations. Previously designed 9-channel Z-gradient coil array and home-built gradient amplifiers (50 V and $20 \mathrm{~A}$ ) are used in the experiments. Three sets of currents optimized for linear Z-gradient, second-order Z2, and third-order Z3 fields are used in the bench-top experiments. The current weightings for the linear Z-gradient are also used as the readout gradient in the 3T MRI experiments.

Results: Current measurements for the example magnetic field profiles with minimum rise times are demonstrated for the simultaneous use of 9-channel gradient coils and amplifiers. MRI experiments verify that a linear Z-gradient field with a desired time waveform can be generated using a mutually coupled array coils.

Conclusion: Bench-top and MRI experiments demonstrate the feasibility of the proposed circuit model and analytical formulas to drive the mutually coupled gradient coils.

\section{K E Y W O R D S}

feedforward model, gradient arrays, gradient power amplifiers, MRI, mutual coupling, mutual inductance, mutually coupled gradient array coils

\section{1 | INTRODUCTION}

In conventional MRI, linear gradients are used to encode the spatial coordinates of spins onto the resonance frequency. In the past decade, gradient coil arrays with

Koray Ertan and Soheil Taraghinia contributed equally to this work. arbitrary magnetic field profiles have proven to be useful for encoding purposes ${ }^{1-4}$ at the expense of increased hardware complexity. Conventional linear gradient coils are generally designed to have minimal coupling, which is not the case for generalized array of coils. High-channel-count gradient elements can couple to each other because of the nonorthogonal magnetic field profiles. Gradient array 
elements are likely to be mutually coupled even for orthogonal spherical harmonics field profiles, ${ }^{5}$ although there have been some efforts to reduce ${ }^{3}$ or avoid mutual coupling between the channels. ${ }^{6}$ On the other hand, driving mutually coupled gradient array coils is possible and might even be preferable to avoid the mutual coupling constraints in the coil design.

Providing high-fidelity and high-bandwidth currents for gradient array systems might be a challenge because of the mutual coupling. A conventional gradient power amplifier (GPA) with a feedback control might help to compensate the effect of induced voltage on the coil due to the mutual coupling. However, required voltages to fully compensate the effect of mutual coupling should be known while designing the gradient current waveforms; otherwise, amplifier voltage limitations might be exceeded and full compensation cannot be realized. Therefore, a feedforward system enabling an analytical calculations of the required voltages might be desirable to drive the mutually coupled gradient array coils.

Gradient array systems should be considered as multiple-input-multiple-output (MIMO) systems, because the time-varying current in 1 element can change the current waveforms of the other elements. If the system is assumed to be linear and time invariant, the frequency response of the system, including the cross terms, can be measured. Once the system is known, appropriate predistortions can be applied to the input waveforms ${ }^{5,7}$ to obtain the desired gradient waveforms. Inverting the frequency response of the system at a high bandwidth might be computationally expensive for a high number of channels and lacks an analytical expression that can be used to calculate the amplifier limitations.

This study is an extended version of a previously presented conference abstract on driving mutually coupled gradient array system. ${ }^{8}$ Here, a first-order time domain model of driving mutually coupled array elements is developed and allows analytical calculation of the required input voltages for a given set of desired output currents. This analytical expression can be used to find the minimum achievable rise time for a given current combination of the coil arrays under the amplifier voltage limitations. The self- and cross-impedances of each element according to the proposed model are compared with impedance measurements in the operating bandwidth of the amplifiers $(<10 \mathrm{kHz})$. The first-order model and expression for the minimum achievable rise time is validated in bench-top experiments with 3 different current combinations that generate a linear Z-gradient and second- and third-order z-shim fields on a 9-channel low-cost home-built gradient array system. Finally, a driving mutually coupled gradient system is demonstrated with MRI experiments.

\section{\begin{tabular}{l|l}
2 & THEORY
\end{tabular}}

\section{1 $\mid$ Circuit model}

Figure 1 shows the linear and time invariant circuit model of the gradient coils and a single stage of the LC gradient filter, where $Z_{L}$ and $Z_{C}$ are the impedances of the series inductor and parallel capacitor of the filter, respectively. Moreover, each cable of the coil is carried inside the MR room using a feedthrough capacitor to filter out the RF noise penetrating into the shielded room. Although capacitance of the feedthrough filters can be added to $Z_{C}$ in differential mode, their impedance is dominated by $Z_{C}$ in the operating bandwidth of the GPAs. Therefore, the capacitance of the feedthrough filter is excluded from the model. The impedance of the mutually coupled gradient coils is modeled by a series combination of a diagonal resistance matrix, $\boldsymbol{R}$, and a mutual coupling matrix, $\boldsymbol{M}$, in which the diagonal terms determine the self-inductance and the off-diagonal terms determine the inductive coupling between the channels. ${ }^{8}$ The input and output characteristics of this model at a single frequency can be modeled as a MIMO system to account for cross couplings as in Equation 1:

$$
V(\omega)=\left(Z_{g}+2 Z_{L} E+\frac{2 Z_{L}}{Z_{C}} Z_{g}\right) I(\omega)
$$

where $\boldsymbol{V}$ and $\boldsymbol{I}$ are the vectors for the input voltage and output current of all channels, and $\boldsymbol{E}$ is the identity matrix.

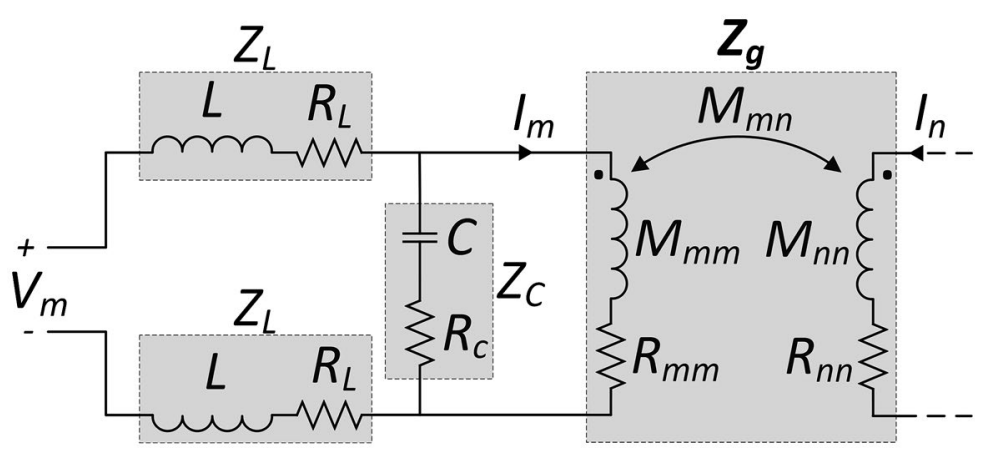

F I G U RE 1 Circuit model for the single-stage LC gradient filters and mutually coupled gradient coils 
$Z_{g}$ is the coil impedance matrix, which can be written as $\boldsymbol{Z}_{g}=\boldsymbol{R}+j \omega \boldsymbol{M}$.

The output filter is designed to suppress the current ripple at the effective switching frequency of the amplifiers while having minimal influence at the bandpass frequencies. The capacitive term in Equation 1 can be neglected in the low-pass regime, which leads to the definition of the first-order time domain relation between the input voltage and output current, as in Equation 2. The first-order time domain expression in Equation 2 is the equation that is used in the pulse width modulation (PWM) calculations throughout the study.

$$
\begin{gathered}
\boldsymbol{V}(t)=\boldsymbol{M}^{\text {total }} \frac{d \boldsymbol{I}(\boldsymbol{t})}{d t}+\boldsymbol{R}^{\text {total }} \boldsymbol{I}(t) \\
\boldsymbol{M}^{\text {total }}=\boldsymbol{M}+2 L \boldsymbol{E} \text { and } \boldsymbol{R}^{\text {total }}=\boldsymbol{R}+2 R_{L} \boldsymbol{E}
\end{gathered}
$$

\section{2 $\quad$ Minimum rise time}

In a mutually coupled array configuration, the maximum amplifier voltage and impedance of a single channel are not sufficient to determine the rise time for a given current value. Current flowing through each coil is highly affected by the currents flowing through the other coils, especially neighboring coils with strong coupling. Therefore, the rise times and resulting slew rates should be calculated for each current amplitude and timing. For a particular time dependence of a fixed magnetic profile, all coils should be driven by currents with different amplitudes but exactly the same time dependence. In the case of a typical trapezoidal current with equal rise and fall times, the peak voltage is required either at the end of the rise period or the beginning of the fall period. If the voltage limitations of the amplifiers are identical for each channel, there is a lower bound on the rise time determined by the coil with the highest peak voltage, which should be driven by the maximum voltage limitation of the amplifiers. Considering Equation 2a, the minimum possible rise time for a trapezoidal time dependence of any magnetic field profile can be calculated as follows:

$$
\Delta t_{\min }=\max _{1 \leq m \leq N}\left\{\frac{\left|\sum_{n=1}^{N} M_{m n}^{\text {total }} \cdot I_{n}\right|}{V_{\text {max }}-\left|R_{m m}^{\text {total }} \cdot I_{m}\right|}\right\}
$$

where $\Delta t_{\min }$ is the minimum rise time, $\mathrm{I}_{\mathrm{n}}$ is the current amplitude of the $n^{\text {th }}$ channel during the plateau period of the trapezoidal waveform, $M_{m n}^{\text {total }}$ and $R_{m m}^{\text {total }}$ are the entries of $\boldsymbol{M}^{\text {total }}$ and $\boldsymbol{R}^{\text {total }}$, respectively, at $\mathrm{m}^{\text {th }}$ row and $\mathrm{n}^{\text {th }}$ column. $V_{\text {max }}$ is the maximum voltage limitation of the identical amplifiers.

\subsection{Optimization of gradient currents}

A gradient array system enables dynamic optimization of the field profile with dynamically adaptable current weightings.
A least squares optimization problem is formulated to solve the optimal current weightings for a given target magnetic field profile, as shown in Equation 4:

$$
\min _{\boldsymbol{I}_{w}, \alpha}\left\|\boldsymbol{B}_{\text {target }}-\alpha \boldsymbol{B} \boldsymbol{I}_{w}\right\|_{2}
$$

where $\boldsymbol{B}_{\text {target }}$ is a column vector consisting of a target magnetic field at discrete locations, $\boldsymbol{B}$ is a matrix with column vectors consisting of measured or simulated magnetic field profiles for a unit current applied to each channel, $\boldsymbol{I}_{\boldsymbol{w}}$ is a column vector representing the current weightings of all channels with unit infinity norm and $\alpha$ is a normalization factor to ensure that current weightings are normalized such that maximum current among the channels are unity. Although one can define many other optimization problems, this optimization problem is preferred for the current weightings in this example because it provides the minimum error norm as the simple analytical solution, as shown in Equation 5:

$$
\boldsymbol{I}_{w}^{*}=\frac{\boldsymbol{B}^{+} \boldsymbol{B}_{\text {target }}}{\left\|\boldsymbol{B}^{+} \boldsymbol{B}_{\text {target }}\right\|_{\infty}}
$$

where $\boldsymbol{B}^{+}$is the pseudoinverse of $\boldsymbol{B}$.

\section{3 | METHODS}

\section{1 | Gradient array system}

Gradient array systems consist of multiple coils driven by individual amplifiers. Figure 2 depicts the main components of the proposed gradient amplifier array setup. Each GPA is used in a 1-stage H-bridge configuration, and a bootstrap circuit is used to drive the high side switches. An isolated gate driver (ADuM7234, Analog Devices, Norwood, MA) is used to switch NMOS transistors (IRFP250n, Infineon Technologies Americas Corp., El Segundo, CA), providing $50 \mathrm{~V}$ and $20 \mathrm{~A}$ at the output. A single power supply is used to feed all the GPAs, because each GPA consists of a single-stage $\mathrm{H}$-bridge rather than a conventional stack configuration. ${ }^{9}$ Component values and models are provided in Supporting Information Table S1, which is available online. Images of the home-built GPAs are shown in Figure 3A.

The evaluation board of a Xilinx Virtex5 FPGA (XMF5, PLDkit OU) with a $100 \mathrm{MHz}$ clock frequency is used to generate the PWM control signals. The PWM period is chosen as $20 \mu \mathrm{s}$, which leads to a $10 \mu$ s dwell time and a 100 $\mathrm{kHz}$ effective PWM frequency for the center-aligned PWM configuration. Considering the clock frequency, the duty cycles can be set approximately with 10 bits of resolution for all channels independently. The FPGA is programmed by a user interface in a PC by means of serial communication. The user interface allows programming of the channel 


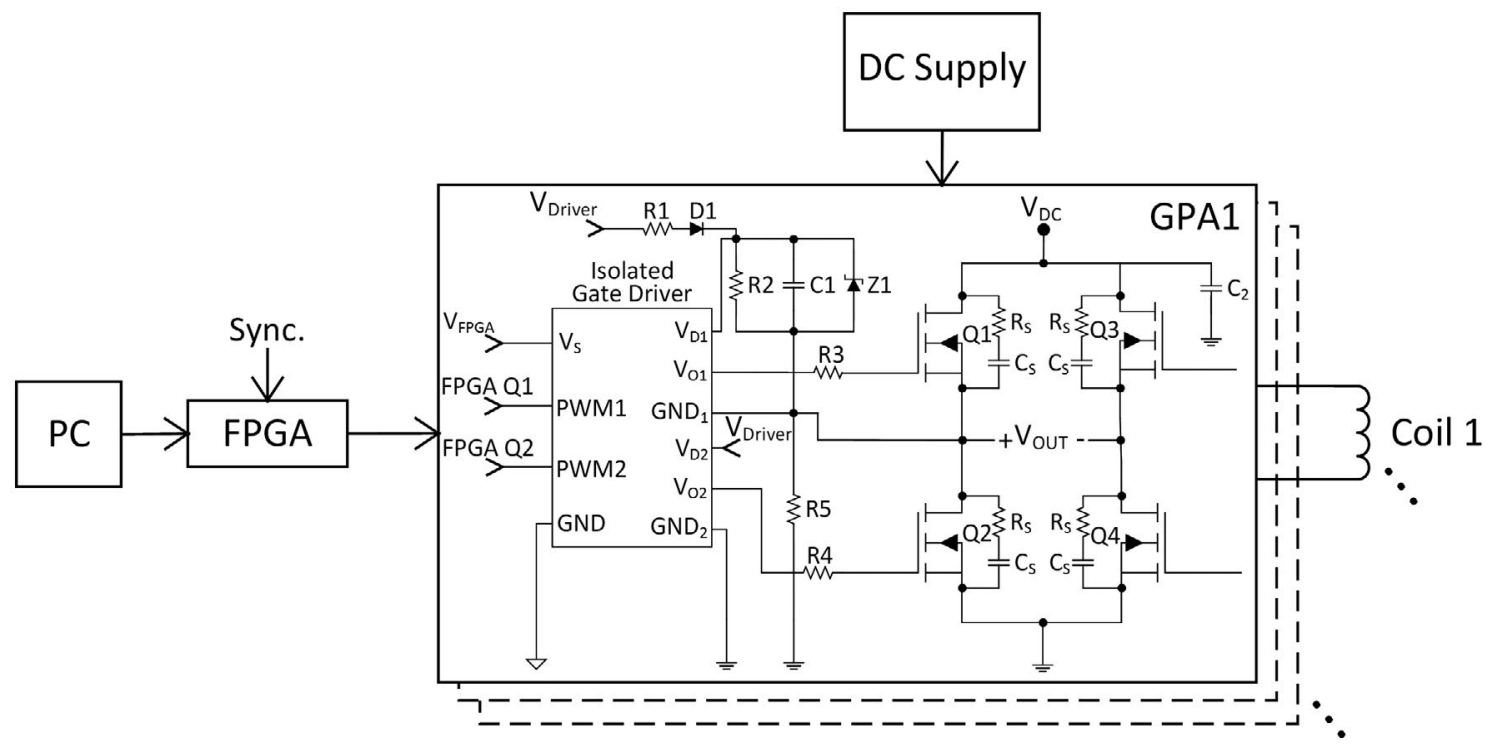

F I G U RE 2 Schematic illustration of the full H-bridge gradient amplifier array (periphery components of the right side are not shown here), including a single power supply, its gate drivers, control signals provided by an FPGA, and gradient coils as loads

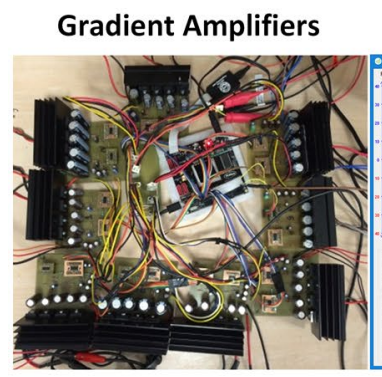

(A)

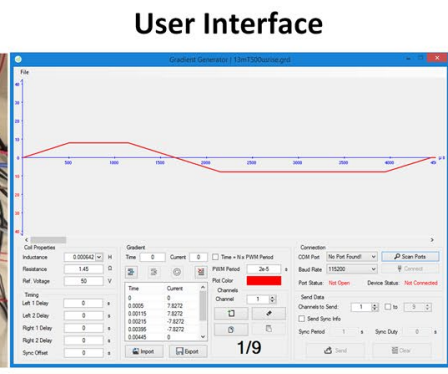

(B)
Coil Array Schematic Ch\#: 123456789

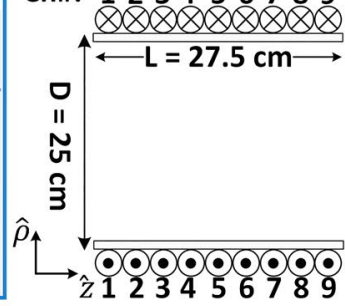

(C)
Gradient Coil Array

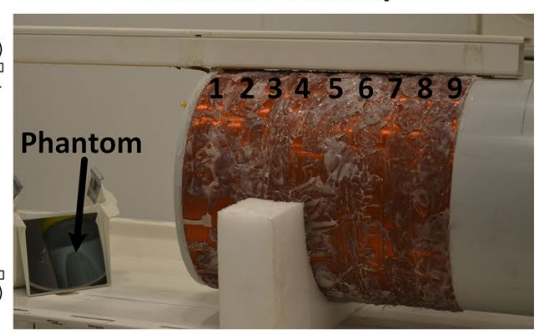

(D)

F I G U RE 3 Nine gradient amplifiers and an FPGA (A), user interface to program the waveforms for all channels (B), schematic illustration of the Z-gradient array $(\mathrm{C})$, and photograph of the 9-channel Z-gradient coil array (D)

impedance, desired current waveforms, PWM delays to prevent shoot through in the H-bridge circuitry, supply voltage, and PWM periods independently for an arbitrary number of channels. A screenshot of the user interface is provided in Figure 3B. The PWM duty cycle calculations are performed using the FPGA based on the MIMO feedforward model proposed in Equation 2. Although combination of the feedforward and feedback controllers might increase the time fidelity of the currents, the required hardware and controller for feedback loop are not realized in this study and left as a future study.

A previously designed 9-channel Z-gradient array is used to demonstrate the proposed method of driving mutually coupled gradient coils. Each gradient channel consists of 36 turns wound on a plastic cylindrical shell with a diameter of $25 \mathrm{~cm}$ and a total length of $27.5 \mathrm{~cm}$ for 9 channels. Tx/Rx birdcage coil and its shield are also placed inside the gradient coil array. Schematic illustration and photograph of the Z-gradient array are provided in Figure $3 \mathrm{C}$ and D, respectively.
More detailed information about the gradient array coil can be found in Ertan et al. ${ }^{1}$

\subsection{Impedance measurements}

The lumped element circuit model assumes that the inductance, capacitance and resistance values are constant in the operating bandwidth of the amplifiers. However, gradient waveforms require high fidelity to the programmer's design. Therefore, the impedance of the filter components and gradient coils are measured as a function of frequency using a GW Instek LCR-B105G high-precision LCR meter (Good Will Instruments, Taiwan) for comparison with the low-frequency approximation of the lumped element circuit model. Extended open and short circuit tests are performed to measure the self and leakage inductance of the mutually coupled coils including the winding resistances. ${ }^{10}$ First, the impedance of the filter components and the self-impedance of each gradient channel are measured up to the operating 
bandwidth of the amplifiers $(\leq 10 \mathrm{kHz})$ with a $100 \mathrm{~Hz}$ step size. Calculation of the frequency-dependent cross-channel impedance, $Z_{m n}$, requires another impedance measurement of the $\mathrm{m}^{\text {th }}$ channel when the $\mathrm{n}^{\text {th }}$ channel is a short circuit. A detailed explanation can be found in Supporting Information Text S1. After calculating the $\boldsymbol{Z}_{g}$, the impedances of the filter components are also measured to calculate the frequency response of the circuit, $V_{m}(\omega) / I_{n}(\omega)$, based on the impedance measurements according to Equation 1.

\section{3 | Experiments}

Driving mutually coupled gradient array system is illustrated with 3 example current vectors optimized for different magnetic field distributions, such as a linear Z-gradient $\left(B_{z} \propto z\right)$, second-order Z2 $\left(B_{z} \propto z^{2}-0.5\left(x^{2}+y^{2}\right)\right.$ and third-order Z3 $\left(B_{z} \propto 2 z^{3}-3 z\left(x^{2}+y^{2}\right)\right)$ fields inside a 15 -cm diameter spherical volume. All computations are performed in MATLAB 2017a (The MathWorks, Natick, MA). Magnetic field map measurements from the previous study ${ }^{1}$ are used to obtain the target magnetic field distribution.

Although full mutual impedance matrix measurements of the 9-channel coil are available, self-inductances and resistances are tuned manually to include the effect of the feed cables and amplifier-related parameters, such as stray inductances and drain source on-resistance of the MOSFETs. Currents flowing through 9 coil elements are captured for the example cases to demonstrate the effect and feasibility of compensating for the mutual coupling between channels. Current measurements are performed on the lab bench using a current probe (LFR06/6 Rogowski Current Waveform Transducer, Power Electronic Measurements Ltd., Nottingham, UK) and a digital oscilloscope (Agilent DSOS104A, Keysight Technologies, Santa Rosa, CA) before moving on to the imaging experiments inside the scanner. Current waveforms are digitized at a rate of $2 \mathrm{MSa} / \mathrm{s}$ to filter out the higherfrequency interference signals in the environment.

Furthermore, MRI experiments are conducted to image an orange as well as a home-built, homogeneous cylindrical phantom with a diameter of $10 \mathrm{~cm}$ that consists of $\mathrm{CuSo}_{4}$ solution at a concentration of $15 \mathrm{mM} / \mathrm{L}$. Central coronal slices (X-Z plane) are imaged using a gradient echo sequence with an field of view of $150 \mathrm{~mm}$, an isotropic in-slice resolution of $1 \mathrm{~mm}$ and a slice thickness of $5 \mathrm{~mm}$. For the orange and phantom images, the echo time/repetition time values are 10/100 $\mathrm{ms}$ and 7/300 ms, respectively. During the experiments, the system Z-gradients are turned off. Coil elements with current weightings optimized for a linear Z-gradient with a gradient strength of $13 \mathrm{mT} / \mathrm{m}$ are used as the prephaser and readout gradients. The prephaser and readout gradients take a total of $1150 \mu$ s and $2300 \mu$ s, respectively, including the rise and fall times of $500 \mu \mathrm{s}$. The maximum gradient amplifier voltage is limited to $40 \mathrm{~V}$, and $80 \%$ of the actual specification of the amplifier increases the reliability of the system.

\section{\begin{tabular}{l|l}
4 & RESULTS
\end{tabular}}

\subsection{Impedance model comparison}

Impedance measurements are performed to test the validity of the first-order lumped element circuit model in the low-pass frequency regime, which is expressed in Equation 2. For the first-order circuit model, inductance values measured at $1 \mathrm{kHz}$ are assumed to be valid over the entire frequency range, and the resistance values measured at DC are used in the entire bandwidth. Figure 4 shows the mutual inductance matrix $(\boldsymbol{M})$ measured at $1 \mathrm{kHz}$ and DC resistance matrix $(\boldsymbol{R})$ of the gradient array coils. The mean value of the self-inductance of the coils is $608 \mu \mathrm{H}$ and the mean value of the mutual inductance between the closest neighboring channels is $328 \mu \mathrm{H}$, which is more than the half of the coil's self-inductance. Mean value of the DC resistance is $1.13 \Omega$. Furthermore, inductance and resistance of the filter inductor are measured as $36 \mu \mathrm{H}$ and $40 \mathrm{~m} \Omega$, respectively, which is also included in the first-order lumped element circuit model. The entire frequency response of the firstorder lumped element circuit model and impedance measurements are compared in Figure 5. An impedance matrix

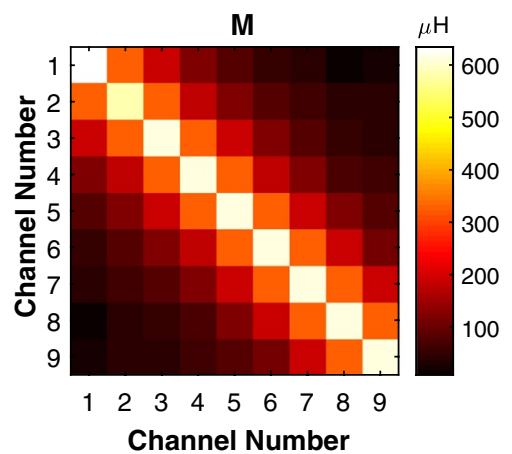

(A)

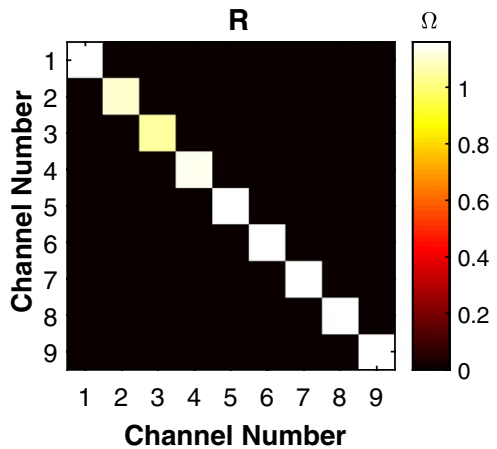

(B)

F I G U R E 4 Mutual inductance matrix, $\boldsymbol{M}$, measured at $1 \mathrm{kHz}$ (A) and DC resistance matrix, $\boldsymbol{R}$, for 9 channel Z-gradient array coils (B) 


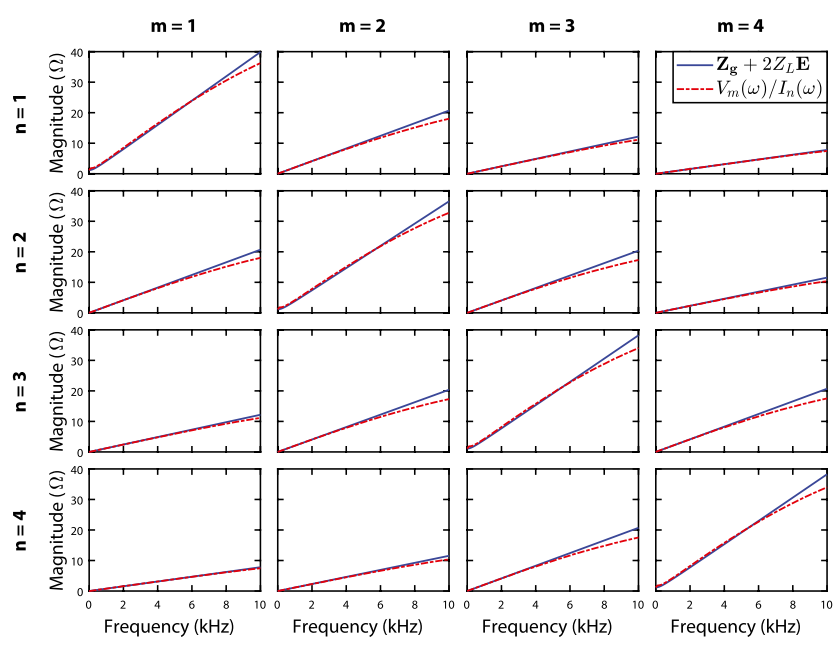

(A)
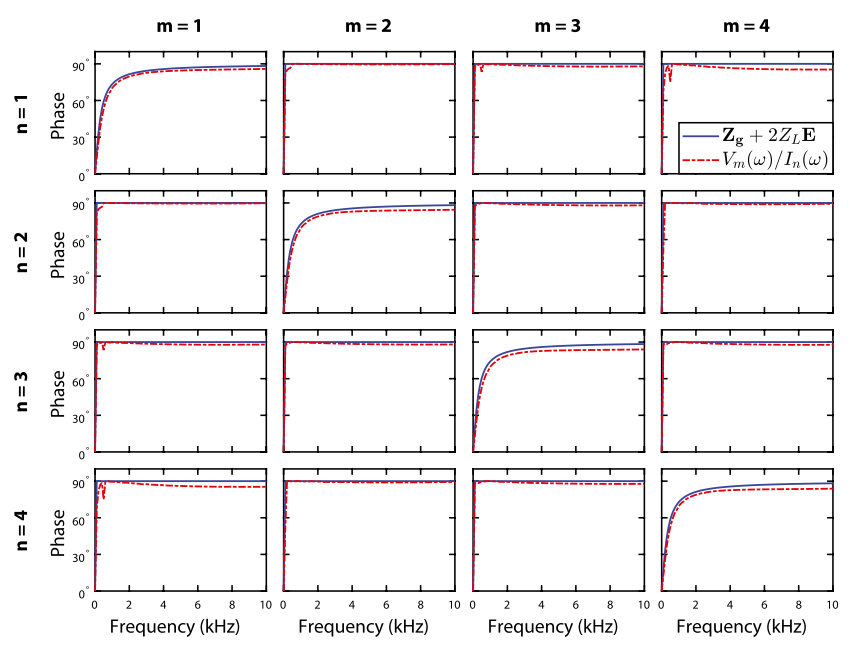

(B)

F I G U R E 5 Impedance matrices are shown for (blue) the proposed circuit model in Equation 2 assuming frequency-independent circuit elements and (red) the calculated ratio of the input voltage for the $\mathrm{m}^{\text {th }}$ channel and output gradient current for the $\mathrm{n}^{\text {th }}$ channel using impedance measurements of coil arrays with filters as a function of frequency. Both the magnitude (A) and phase (B) of the impedance matrices are shown

for 4 representative channels is shown, and similar characteristics are present for the other channels.

The impedance measurements can be used to analyze the assumptions made in Equation 2. Each coil element is modeled as an inductance and a DC resistance by assuming that (1) there is no AC resistance; and (2) the self-resonance frequency of the coil is at much higher frequencies than the operating bandwidth of the amplifiers; the effect of the filter capacitor in Equation 1 is also neglected in Equation 2. Based on these assumptions, magnitude of the impedances increases linearly as a function of frequency with a slope proportional to the corresponding self or mutual inductance. These assumptions lead to only a $1 \%$ deviation in the self-impedance on average at $5 \mathrm{kHz}$ compared with the frequency response measurements. For frequencies greater than $5 \mathrm{kHz}$, the capacitive effects become notable, and the circuit model overestimates the selfimpedance by nearly $12 \%$ for all channels at $10 \mathrm{kHz}$. The average phase error of the self-impedance is $4^{\circ}$ at $10 \mathrm{kHz}$, which originates mostly from the increase in the AC resistance of the coil. Moreover, the circuit model also underestimates the amplitude of the mutual impedances at frequencies higher than $5 \mathrm{kHz}$ due to capacitive effects. The average phase of the mutual impedance between any channel and the closest neighbor is $88^{\circ}$ at $10 \mathrm{kHz}$. The phase of the mutual coupling might deviate from $90^{\circ}$ due to the losses during the coupling. Therefore, the purely inductive mutual coupling assumption in Equation 2 causes a $2^{\circ}$ phase error in the frequency response of the cross terms.

\section{2 $\quad$ Compensation of mutual coupling}

In highly coupled gradient array coils, the actual current waveforms might significantly deviate from the desired current waveform if mutual coupling is not compensated during the voltage calculations. The current error might be in different forms depending on the individual current amplitudes and waveforms for the mutually coupled coils, as illustrated in Figure 6. When the self-inductances of 2 coils are comparable and the mutual coupling coefficient is positive, the current flowing in the same direction, depending on their amplitudes, might lead to an undershoot and increase the rise time of the desired current for both channels because of the opposing induced voltage (Figure 6A). Similarly, reverse directed currents flowing in the 2 coils can cause an overcurrent in both channels (Figure 6B). Moreover, even the direction of the current might change from the desired current direction if the induced voltage is greater than the applied voltage (Figure 6C). In particular, the current waveforms of channels with relatively lower desired current values might be significantly influenced by the channels with higher current values, as shown in Figure 6C. When the mutual coupling is considered as in Equation 2, desired current waveforms can be achieved with significantly less error. Therefore, mutual coupling should be considered for the accurate generation of mutually coupled coil currents.

\section{3 | Benchtop experiments}

The current weightings are optimized for 3 example magnetic field profiles, such as the Z, Z2, and Z3 fields, using Equation 5. The optimal current weightings are scaled by $20 \mathrm{~A}$ when the amplifiers are driven at their maximum current limits. The target magnetic fields and the obtained magnetic field profiles using the superposition of the previously measured magnetic field map of each channel with optimized current weightings and error between the target and obtained magnetic field profiles are provided in Figure 7. The optimized linear Z-gradient field profile is also used as a readout gradient during the experiments. 
Furthermore, the minimum attainable rise times for the optimized current vectors are calculated using Equation 3 as 450 $\mu \mathrm{s}, 440 \mu$ s, and $380 \mu$ s for the $\mathrm{Z}, \mathrm{Z} 2$, and Z3 fields, respectively. The user interface of the gradient amplifiers is programmed to provide trapezoidal current waveforms with minimum rise

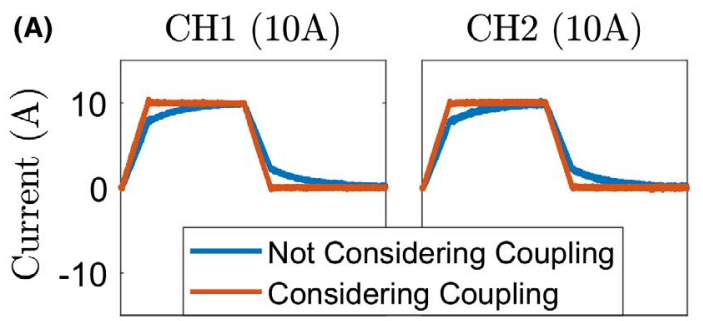

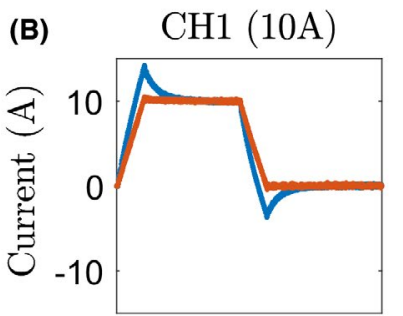

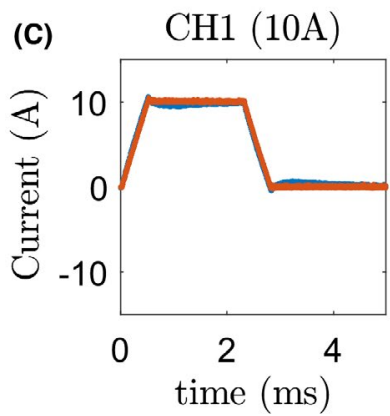

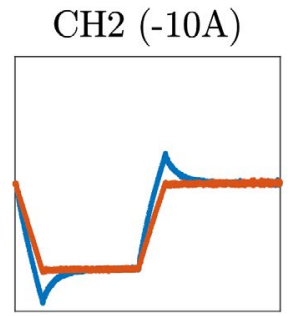

$\mathrm{CH} 2(2 \mathrm{~A})$

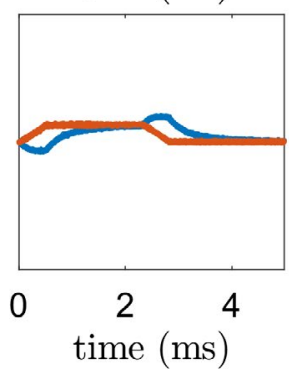

F I G URE 6 Experimental results for the current measurements of 2 channels when mutual coupling is or is not compensated during the input voltage calculations. Three example current pairs are demonstrated for $\mathrm{Ch} 1$ and $\mathrm{Ch} 2$ as $10 \mathrm{~A}$ and $10 \mathrm{~A}(\mathrm{~A}), 10 \mathrm{~A}$ and $-10 \mathrm{~A}$ (B), and $10 \mathrm{~A}$ and $2 \mathrm{~A}(\mathrm{C})$ times. Figure 8 shows the measured current waveforms passing through all gradient channels for the 3 field profiles. Small current drops were observed in the plateau region of some of the trapezoidal waveforms, most likely because of the supply voltage drops during high-demand use. Moreover, there is no feedback loop in the amplifiers to ensure that the output current accurately tracks the desired input signal. However, all current waveforms increased to the desired value in the minimum possible rise time, which confirms the circuit model in Equation 2 and the analytical expression in Equation 3.

For each desired current vector, required voltage for each channel is calculated using Equation 2a. The required voltage

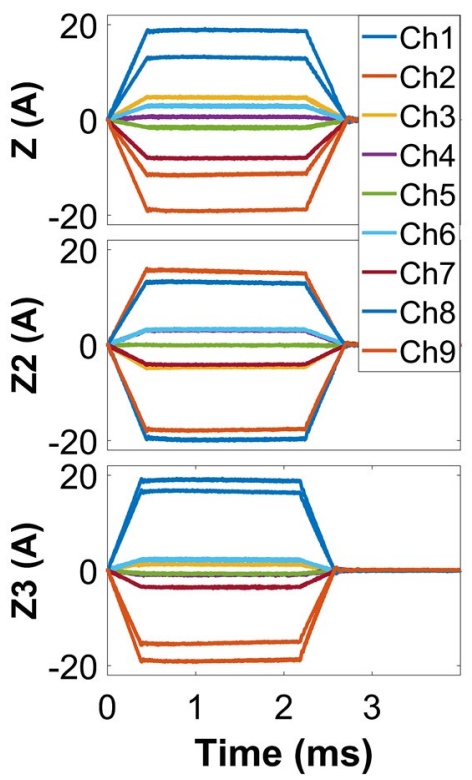

F IG URE 8 Current measurements of 9 channels for the Z, $\mathrm{Z} 2$, and Z3 field profiles. Trapezoidal current waveforms with analytically calculated minimum rise times are applied to all channels simultaneously
F I G URE 7 Magnetic field profiles for linear Z $\left(\boldsymbol{B}_{z} \propto z\right)$, second-order Z2 $\left(\boldsymbol{B}_{z} \propto z^{2}-0.5\left(\boldsymbol{x}^{2}+\boldsymbol{y}^{2}\right)\right)$ and third-order Z3 $\left(\boldsymbol{B}_{z} \propto 2 z^{3}-3 z\left(\boldsymbol{x}^{2}+\boldsymbol{y}^{2}\right)\right)$ magnetic fields. Row 1, Target magnetic field profiles. Row 2, Superposition of measured magnetic field profiles with optimized current weightings, that is, optimized field profiles. Row 3, Error maps between the target and optimized magnetic field profiles. The red circles indicate the optimization box, which is a $15 \mathrm{~cm}$ diameter spherical volume
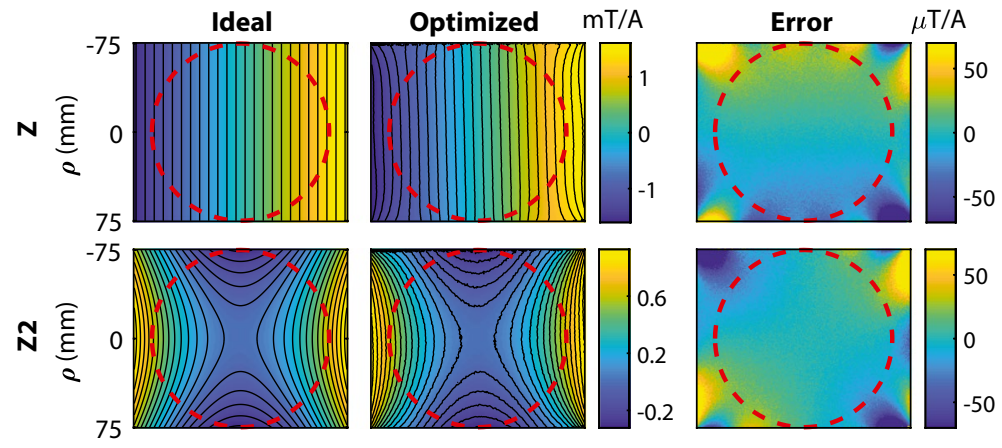

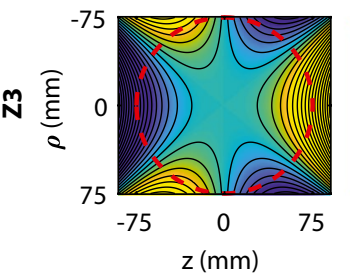

(A)

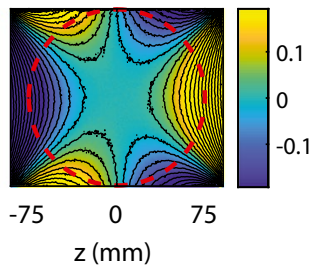

(B)

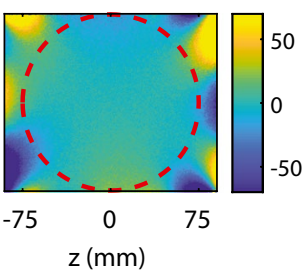

(C) 
for each channel can be decomposed into 3 different voltages such as $V_{R}, V_{\text {Self }}$, and $V_{\text {Coupling }}$ for the interpretation purposes. $V_{R}$ represents the voltage required by the resistive part of the impedance matrix $\left(\boldsymbol{R}^{\text {total }}\right) . V_{\text {Self }}$ is the voltage required by the self-inductance of each channel which only accounts for the diagonal entries of $\left(\boldsymbol{M}^{\text {total }}\right)$. Voltage induced on each coil due to the cross couplings is represented by the $V_{\text {Coupling }}$, which is determined by the off-diagonal entries of the $\boldsymbol{M}^{\text {total }}$, current flowing through all other channels and rise/fall time of the trapezoidal waveform. In other words, $V_{\text {Coupling }}$ represents the amount of voltage that should be considered to account for the couplings. Summation of these 3 decomposed voltages results in the applied voltage to the corresponding coil element. Figure 9 shows the decomposed drive voltages of each channel for 3 current vectors in Figure 8. Because peak voltage for each channel is reached either at the end of the rise period or at the beginning of the fall period, voltage values are provided at the end of the rise period in Figure 9. At the beginning of the fall time, signs of the $V_{\text {Self }}$ and $V_{\text {Coupling }}$ should be reversed. In Figure 9, it can be observed that $V_{\text {Coupling }}$ can be as high as $V_{\text {Self }}$ for some channels. It should also be noted to that $V_{\text {Coupling }}$ and $V_{\text {Self }}$ have opposite signs and coupling decreases the demand on the voltage waveforms for these specific examples only because direction of the current incidentally alternates between the neighboring channels for least square field errors. For the general case, $V_{\text {Coupling }}$ and $V_{\text {Self }}$ can be destructively or constructively superposed depending on the direction of the currents as already shown in Figure 6.

\subsection{MRI experiments}

Figure 10 shows a comparison of the coronal MRI images acquired using a conventional Z-gradient system and the $\mathrm{Z}$-gradient generated by the array system as a readout gradient. There are no significant spatial distortions in the images
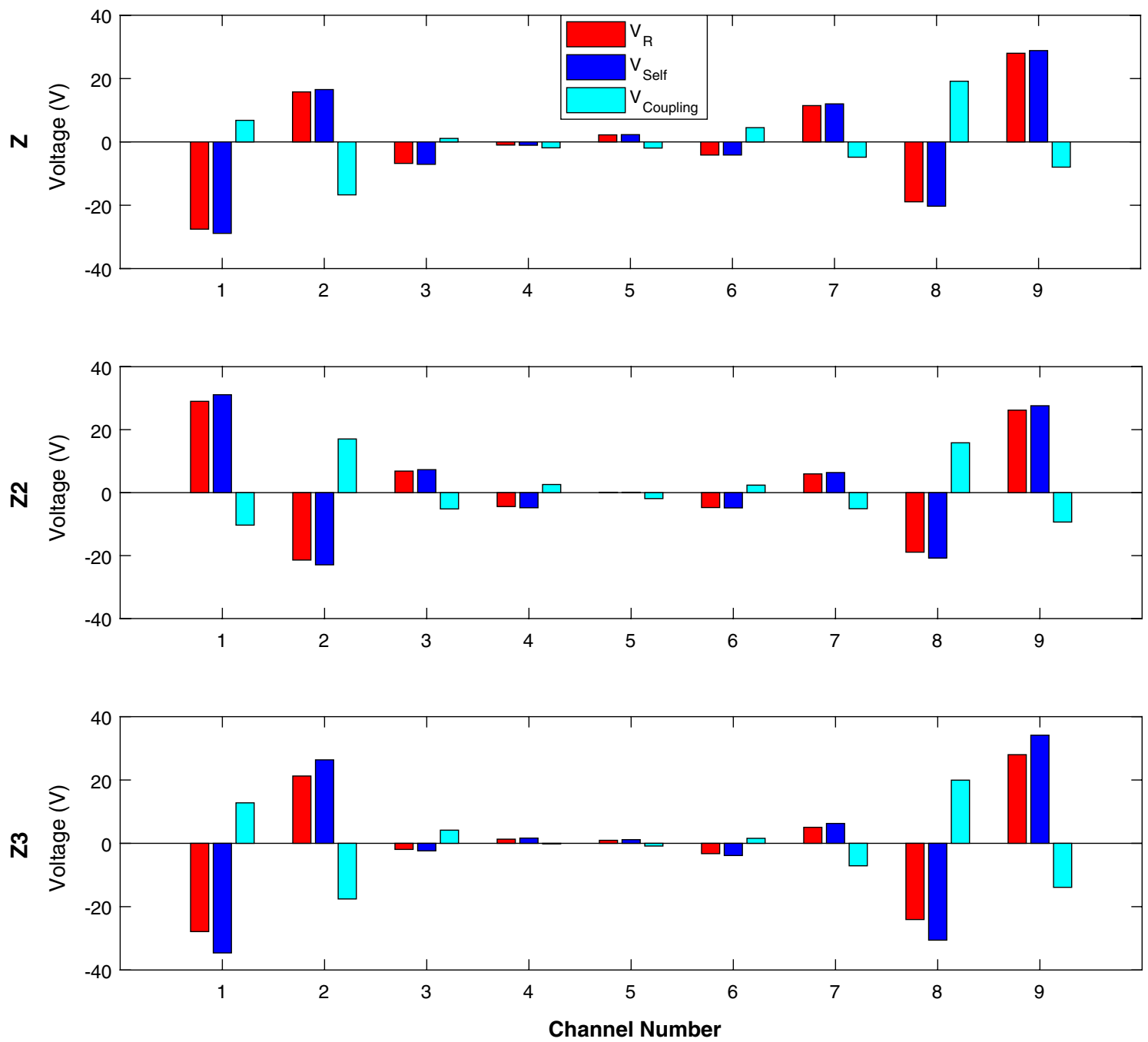

F I G U RE 9 Decomposed drive voltage amplitudes, $V_{R}, V_{\text {Self }}$, and $V_{\text {Coupling }}$, at the end of the rise period of the trapezoidal current waveforms in Figure 8. For the voltages at the beginning of the fall period, signs of both $V_{\text {Self }}$ and $V_{\text {Coupling }}$ should be reversed. It should also be noted that applied coil voltages can be calculated by summing up the contributions of $V_{R}, V_{\text {Self }}$, and $V_{\text {Coupling }}$ 


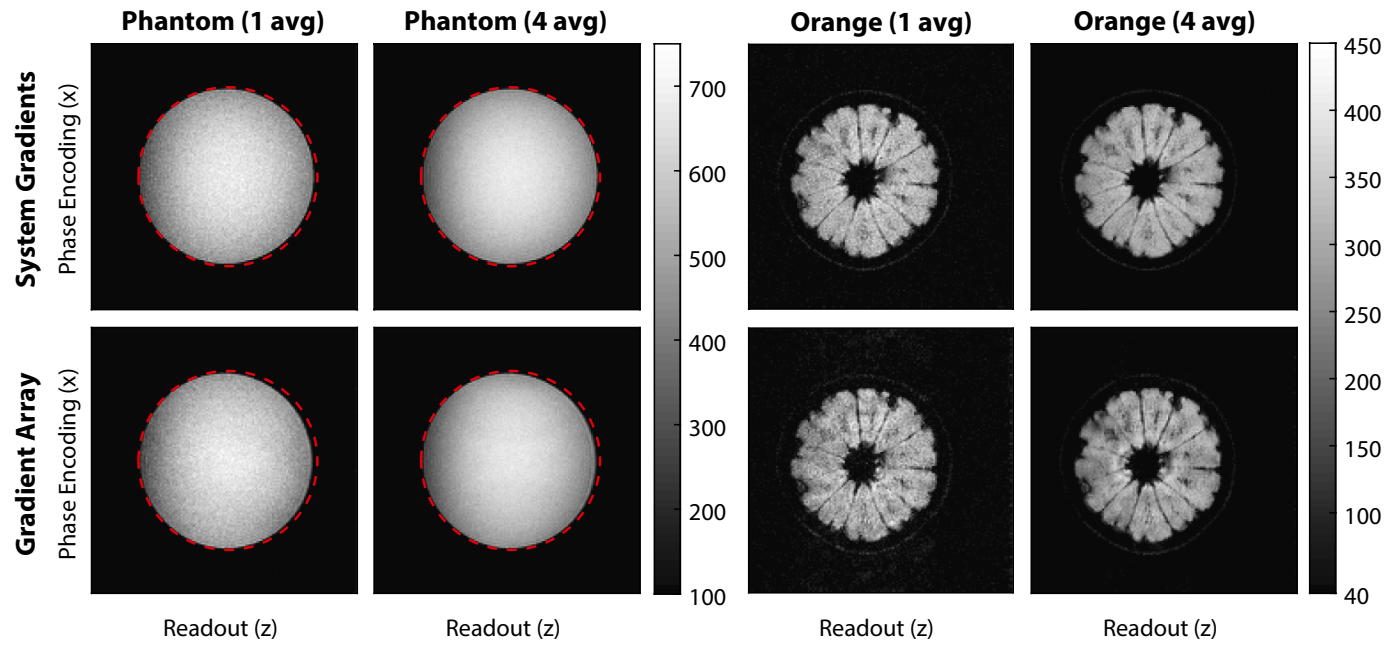

F I G URE 10 Coronal MRI images of a cylindrical phantom and an orange with 1 and 4 averages. Row 1. The system Z-gradient. Row 2, Gradient array system is used as the readout gradient for comparison. The fixed red circles indicate the boundaries of the phantom

of the cylindrical phantom, which validates 2 claims. First, the linearity of the magnetic field generated by the array system is comparable to the linearity of the conventional gradient coil. Second, the desired gradient moment is achieved between the readout samples, because the dimensions of the phantoms are almost identical. Additionally, both the phantom and orange are imaged using an averaging factor of 4 to test the repeatability of the experiments and increase the SNR of the images. The agreement between the images taken with the system gradients and the gradient array can be considered as a proof of concept for the proposed method of driving mutually coupled array coils. Furthermore, using a gradient array system as a readout gradient results in some artifacts in the phase encoding direction, which can be visually inspected in Supporting Information Figure S2.

\section{5 | DISCUSSION}

\subsection{Effect of the gradient filter}

The impedance measurements of the self-impedance and mutual impedance of the coil alone are in good agreement with the model in which each coil is represented by a series RL circuit and inductively coupled with other series RL circuits (see Supporting Information Figure S1). Although these results are specific to our custom-designed Z-gradient array coil, the technique is proposed for general gradient coil arrays based on the fact that the self-resonance frequency of the gradient coils is designed to be much higher than the switching frequency of the amplifiers. However, the frequency response between the amplifier's output voltage and the coil current can be affected by the capacitive components of the gradient filter. Because the firstorder model overestimates the impedance of the system as in Figure 5, the channel voltages in Equation 2 and the minimum rise time in Equation 3 are also overestimated. Therefore, neglecting the capacitive effects in analytical models does not pose a risk of exceeding the amplifier voltage limitations. Filter effects are apparent toward the end of the bandwidth, which generally contains lower power. Furthermore, higher-order filters can be designed with sharper frequency responses to be transparent in the operating bandwidth of the coil currents while providing enough suppression at the switching frequency. ${ }^{11,12}$ In this study, a single-stage LC filter is preferred to avoid a further increase in the complexity of the hardware. Higher-order filters might increase the physical space requirements, power dissipation and cost of the hardware.

\section{$5.2 \mid$ Feedback requirement}

In this study, the gradient array system is modeled as an linear and time invariant system and included as a MIMO feedforward model in the digital controller of GPAs. Although this model provides currents to the coils with adequate accuracy to perform the imaging, higher-order effects and nonlinear effects such as thermal effects, power source and amplifier imperfections can still degrade the time fidelity of the coil currents. ${ }^{5}$ Therefore, a closed loop feedback system might be required to compensate for the residual current errors. An ideal gradient amplifier with infinite voltage compliance and ideal feedback loop might also compensate the effect of the mutual coupling without having the feedforward model. However, realistic GPAs with voltage limitations might fail to fully compensate the effect of strong mutual coupling if required compensation voltage are not considered while designing the gradient current waveforms. Therefore, feedforward model is still desirable because it enables analytical calculations of the required voltages and the minimum achievable rise times. The design of the current controller for 
the mutually coupled coil arrays might also require multivariable feedback control ${ }^{13}$ because of the cross-talk between the channels, which requires further investigation. The firstorder model in combination with a single-input-single-output feedback loop might provide the desired waveforms with lower computational complexity as an alternative to a full frequency response correction of the system. ${ }^{5,7}$

Determination of the circuit model parameters is prone to measurement errors. Model parameters might also be time varying due to heating related problems or mechanical changes in the system. A feedback loop can be useful to compensate the errors in the model parameters. Furthermore, model parameters including only the coil elements and gradient filters are measured in this study; however, manual tuning is still necessary to account for the effect of feed cables and amplifier related parameters. In a high channel count array system, manual tuning of the parameters might not be practical. It would be beneficial to use the GPAs itself as an auto-calibration system that can extract the model parameters including the effect of GPAs on the impedance. Similar to a feedback loop, an auto-calibration system can be used dynamically to track the time varying changes in the model parameters. Auto-calibration system might use extended open and short circuit test as in this study as well as different coupling measurement techniques. Series-aiding series-opposing method ${ }^{10}$ might not be practical because it requires electrical switches between all combination of array elements for autocalibration. Voltage based measurement techniques can be used to characterize the method; however, high frequency switching noise on the voltage should be handled.

\section{3 | Eddy current compensation}

Although our coil array is not shielded, eddy current effects are negligible due to the small radius of the coils. Each element can be designed to be actively self-shielded ${ }^{3}$ or a shield can be designed as an array of coils driven by independent amplifiers. The proposed model is very similar to the conventional eddy current compensation method, which assumes multiple couplings between the conductive surfaces and the gradient coils. ${ }^{14}$ Recently developed methods of eddy current preemphasis calculations of coupled shim coils ${ }^{15}$ can also be used together with the proposed method to account for the couplings with both passive eddy current surfaces and other actively driven coupled coils. Moreover, there are some programmable pre-emphasis systems that can adapt the pre-emphasis coefficient dynamically, ${ }^{16}$ which can be adapted for mutual coupling compensation dynamically as part of the spectrometer.

\section{4 | Hardware perspective}

Significant voltages can be induced on the loads due to the high change rate of the currents in the neighboring channels.
Therefore, voltage ratings of the amplifier components should be considered at the design stage to ensure that no component is at risk during the worst-case coupling scenarios. As another extreme case, induced voltages might even exceed the supply voltage of the amplifiers, which might cause some unexpected current flow through the freewheeling diodes of the transistors. Furthermore, the malfunctioning of an amplifier might also damage the other amplifiers due to coupling; therefore, the safety precautions should also consider the mutual coupling between channels by monitoring the current and voltage of all amplifiers.

Although extreme couplings might be troubling in terms of the amplifier, mutual coupling might be used to decrease undesired current ripples originating from the switching voltages. Multistage H-bridge amplifiers can accompany phase-shifted PWM schemes ${ }^{17}$ to increase the effective switching frequency, which results in reduced current ripples. Single-stage amplifiers cannot increase the effective switching frequency in their normal operation mode, and optimized relative phases for the PWM signals of the amplifiers along with their mutually coupled loads can decrease the energy of the current ripples by more than $90 \%$ in some cases. ${ }^{18}$ The PWM phase shifting strategy can be used together with gradient filters to further reduce the current ripples. ${ }^{19}$ The advantage provided by the relative phases of the PWM control signals for an amplifier array might also be exchanged for simpler gradient filters with lower series inductance, smaller physical size, lower power dissipation, or lower phase delays.

\section{5 | Applications of coil arrays}

Previously, it was demonstrated that nonorthogonal field profiles can compete with conventional orthogonal spherical harmonic fields for dynamic shimming purposes using a high number of shim array coils ${ }^{20-25}$ or RF coils as shim coils with dedicated hardware. ${ }^{26,27}$ Although the gradient field demands in terms of switching rates and field amplitudes are higher than in shim coils, mutual coupling might still affect the shimming performance of shim coil arrays in theory. Similarly, shim coil arrays are generally used simultaneously with conventional linear gradients, which might induce undesired currents in the shim coils due to mutual coupling between the system gradients and shim arrays. Effect of the mutual coupling on shimming performance depends on several factors such as sequence timings, mutual inductance between the shim array elements and their position relative to the conventional linear gradient coils. More importantly, shim coil arrays can also generate linear gradient profiles ${ }^{2}$ and can be used for combined imaging and shimming applications. ${ }^{28}$ In this case, mutual coupling should also be considered, because the shim array current should be switched at faster rates for imaging purposes. 
Furthermore, some single-channel insert coils with nonorthogonal field profiles might be advantageous to mitigate the $\mathrm{B}_{1}{ }^{+}$inhomogeneity, ${ }^{29}$ field of view reduction, ${ }^{30}$ and diffusion encoding ${ }^{31}$ when simultaneously used with linear gradients. Mutual coupling between the insert coil and the system gradient coils can also significantly degrade the performance of the system depending on the application.

\section{6 | Mutual coupling considerations during the coil design}

Nonorthogonal field profiles generated by arbitrary coil geometries are likely to cause mutual coupling between the channels. Mutual coupling might be reduced in the design stage by physically shifting the coil elements. ${ }^{3}$ Coil arrays can also be designed by constraining the field orthogonality of the channels; however, discretization of the surface currents might still cause some residual mutual coupling. ${ }^{6}$ For both strategies, the aim of avoiding mutual coupling results in more constraints and fewer degrees of freedom in the system design. Furthermore, coil manufacturing, the scarcity of available space on the coil surfaces, the feed cables of the coils and the high number of coil elements can still cause mutual coupling even if it is avoided in the design process. Therefore, the proposed method of modeling and controlling the mutual coupling of gradient array systems might be preferable.

\subsection{Field design flexibility}

Driving each coil element independently enables dynamic gradient design. For conventional gradients, design parameters such as volume of interest, gradient linearity, gradient strength, and the inductance of the coils are specified during the design stage and cannot be changed after manufacturing the coils. In contrast, some tradeoffs between the design parameters can be dynamically used depending on the target volumes and imaging sequences for array systems. The proposed formulation in Equation 3 might be included in the optimization problems for better usage of hardware slew rate limits (i.e., voltage ratings) in exchange for sacrificing other field parameters. However, physiological slew rate limits due to peripheral nerve stimulation are still an open question for gradient coil arrays because the vector E-field distribution generated by each element is dynamically superposed when coil currents are dynamically changing. Therefore, the proposed study enables the analysis and optimization of the slew rate limits in terms of hardware capabilities, while physiological slew rate limits require further investigation.

\section{6 | CONCLUSIONS}

In this study, the mutual coupling of gradient coil arrays is analyzed and modeled using a first-order approximation. The minimum rise time for a given current waveform and the voltage limitations are analytically formulated. The proposed technique is validated with both current measurements and MRI experiments using 9-channel Z-gradient coil array and 9 low-cost home-built gradient amplifiers.

\section{ACKNOWLEDGMENTS}

The authors thank Alireza Sadeghi-Tarakameh for the RF coil production and Hamed Mohammadi for implementing the user interface of the control signals.

\section{REFERENCES}

1. Ertan K, Taraghinia S, Sadeghi A, Atalar E. A z-gradient array for simultaneous multi-slice excitation with a single-band RF pulse. Magn Reson Med. 2018;80:400-412.

2. Juchem C, Nahhass OM, Nixon TW, de Graaf RA. Multi-slice MRI with the dynamic multi-coil technique. NMR Biomed. 2015;28:1526-1534.

3. Littin S, Jia F, Layton KJ, et al. Development and implementation of an 84-channel matrix gradient coil. Magn Reson Med. 2018;79:1181-1191.

4. Ertan K, Taraghinia S, Saritas EU, Atalar E. Local optimization of diffusion encoding gradients using a Z-gradient array for echo time reduction in DWI. In: Proceedings of the 26th Annual Meeting of ISMRM, Paris, France, 2018. Abstract 1641.

5. Vannesjo SJ, Duerst Y, Vionnet L, et al. Gradient and shim preemphasis by inversion of a linear time-invariant system model. Magn Reson Med. 2017;78:1607-1622.

6. Smith E, Freschi F, Repetto M, Crozier S. The coil array method for creating a dynamic imaging volume. Magn Reson Med. 2017;78:784-793.

7. Stich M, Wech T, Slawig A, et al. Gradient waveform pre-emphasis based on thegradient system transfer function. Magn Reson Med. 2018;80:1521-1532.

8. Ertan K, Taraghinia S, Atalar E. Driving mutually coupled coils in gradient array systems in magnetic resonance imaging. In: Proceedings of the 26th Annual Meeting of ISMRM, Paris, France, 2018. Abstract 1756

9. Sabate J, Garces LJ, Szczesny PM, Li Q, Wirth WF. High-power high-fidelity switching amplifier driving gradient coils for MRI systems. Power Electronics Specialists Conference, 2004. PESC 04. 2004 IEEE 35th Annual; 2004: IEEE. p. 261-266.

10. Hayes JG, Cashman D, Egan MG, O'Donnell T, Wang N. Comparison of test methods for characterization of high-leakage two-winding transformers. IEEE Trans Ind Appl. 2009;45: 1729-1741.

11. Sabate J, Schutten M, Steigerwald R, Li Q, Wirth WF. Ripple cancellation filter for magnetic resonance imaging gradient amplifiers. Applied Power Electronics Conference and Exposition, 2004. APEC'04. Nineteenth Annual IEEE; 2004: IEEE. p. 792-796.

12. Yu H, Littin S, Jia F, Kroboth S, Zaitsev M. Output dilter design for gradient amplifier and shimming amplifier of MRI: an overview. In: Proceedings of the 26th Annual Meeting of ISMRM, Paris, France, 2018. Abstract 4428.

13. Skogestad S, Postlethwaite I. Multivariable Feedback Control: Analysis and Design. New York: John Wiley \& Sons; 2007. 
14. Jehenson P, Westphal M, Schuff N. Analytical method for the compensation of eddy-current effects induced by pulsed magnetic field gradients in NMR systems. J Magn Reson. 1990;90:264-278.

15. Fillmer A, Vannesjo SJ, Pavan M, Scheidegger M, Pruessmann KP, Henning A. Fast iterative pre-emphasis calibration method enabling third-order dynamic shim updated fMRI. Magn Reson Med. 2016;75:1119-1131.

16. Gach HM, Lowe IJ, Madio DP, et al. A programmable pre-emphasis system. Magn Reson Med. 1998;40:427-431.

17. Lai R, Sabate J, Chi S, Skeffington W. High performance gradient driver for magnetic resonance imaging system. Energy Conversion Congress and Exposition (ECCE), 2011 IEEE; 2011: IEEE. p. $3511-3515$.

18. Taraghinia S, Ertan K, Atalar E. Minimum current ripple in the gradient array system by applying optimum-phase pulse-width modulation pattern. In: Proceedings of the 25th Annual Meeting of ISMRM, Honolulu, Hawaii, 2017. Abstract 2686.

19. Taraghinia S, Ertan K, Yardim AB, Atalar E. Efficient ripple current reduction in gradient array system using optimized-phase control signals with one stage LC filter. In: Proceedings of the 34th Annual Meeting of ESMRMB, Barcelona, Spain, 2017. Abstract 892.

20. Juchem C, Nixon TW, McIntyre S, Boer VO, Rothman DL, de Graaf RA. Dynamic multi-coil shimming of the human brain at 7 T. J Magn Reson. 2011;212:280-288.

21. Juchem C, Nixon TW, McIntyre S, Rothman DL, de Graaf RA. Magnetic field modeling with a set of individual localized coils. J Magn Reson. 2010;204:281-289.

22. Juchem C, Rudrapatna SU, Nixon TW, de Graaf RA. Dynamic multi-coil technique (DYNAMITE) shimming for echoplanar imaging of the human brain at 7 Tesla. NeuroImage. 2015;105:462-472.

23. Topfer R, Starewicz P, Lo K-M, et al. A 24-channel shim array for the human spinal cord: Design, evaluation, and application. Magn Reson Med. 2016;76:1604-1611.

24. Stockmann JP, Wald LL. In vivo B0 field shimming methods for MRI at 7 T. NeuroImage. 2018;168:71-87.

25. Schwerter $\mathrm{M}$, Moon $\mathrm{CH}$, Hetherington $\mathrm{H}$, et al. Inter-slice current constrained B0 shim optimization for high order dynamic shim updating with strongly reduced eddy currents. In: Proceedings of the 26th Annual Meeting of ISMRM, Paris, France, 2018. Abstract 0836.

26. Han H, Song AW, Truong TK. Integrated parallel reception, excitation, and shimming (iPRES). Magn Reson Med. 2013;70:241-247.

27. Stockmann JP, Witzel T, Keil B, et al. A 32-channel combined $\mathrm{RF}$ and B0 shim array for 3T brain imaging. Magn Reson Med. 2016;75:441-451.
28. Umesh Rudrapatna S, Fluerenbrock F, Nixon TW, de Graaf RA, Juchem C. Combined imaging and shimming with the dynamic multi-coil technique. Magn Reson Med. 2019;81:1424-1433.

29. Ertan K, Atalar E. Simultaneous use of linear and nonlinear gradients for $\mathrm{B} 1+$ inhomogeneity correction. NMR Biomed. 2017;30:e3742.

30. Wiesler DG, Wen H, Wolff SD, Balaban RS. Reduction of field of view in MRI using a surface-spoiling local gradient insert. J Magn Reson Imaging. 1998;8:981-988.

31. Feldman RE, Scholl TJ, Alford JK, Handler WB, Harris CT, Chronik BA. Results for diffusion-weighted imaging with a fourthchannel gradient insert. Magn Reson Med. 2011;66:1798-1808.

\section{SUPPORTING INFORMATION}

Additional supporting information may be found online in the Supporting Information section at the end of the article.

FIGURE S1 Impedance matrices are shown for (blue) the proposed circuit model for the gradient array coils without filters assuming frequency independent circuit elements and (red) measured self and mutual impedances using impedance meter measurements as a function of frequency. Both (A) magnitude and (B) phase of the impedance matrices are shown

FIGURE S2 Coronal MRI images of a cylindrical phantom and an orange with 1 and 4 averages. (row 1) The system Z-gradient and (row 2) gradient array system is used as the readout gradient for comparison. Figure 10 of the main text is reproduced with different color map to highlight the background artifacts. Exact source of the artifact has not been identified. Possible reasons for the artifacts include mechanical vibrations of the setup and/or some amplifier imperfections

TABLE S1 List of the resistor and capacitor values in Figure 2 are shown. The model of D1 is 1N4148 (Vishay Intertechnology Inc., Pennsylvania, USA). The model of Z1 is BZX85C15 (Vishay Intertechnology Inc., Pennsylvania, USA)

How to cite this article: Ertan K, Taraghinia S, Atalar E. Driving mutually coupled gradient array coils in magnetic resonance imaging. Magn Reson Med. 2019;82:1187-1198. https://doi.org/10.1002/mrm.27768 